\title{
Mechanism and Effectiveness of Ti-based Nano-Electrode for Electrochemical Denitrification
}

\author{
Lele Wang ${ }^{1,2}$, Miao $\mathrm{Li}^{2, *}$, Xiang Liu ${ }^{2, *}$, Chuanping Feng ${ }^{1}$, Fang Zhou ${ }^{2}$, Nan Chen ${ }^{1}$, Weiwu Hu ${ }^{l}$ \\ ${ }^{1}$ School of Water Resources and Environment, China University of Geosciences (Beijing), Beijing \\ 100083, China \\ ${ }^{2}$ School of environment, Tsinghua University, Beijing 100084, China \\ *E-mail: watersml@126.com, x.liu@tsinghua.edu.cn
}

doi: $10.20964 / 2017.03 .50$

Received: 2 September 2016 / Accepted: 12 January 2017 / Published: 12 February 2017

\begin{abstract}
A novel Ti-based nano-electrode was fabricated to improve electrochemical nitrate reduction efficiency. The results of scanning electron microscopy (SEM) and X-ray photoelectron spectroscopy (XPS) demonstrated that the surface of the Ti-based nano-electrode was covered with a large number of nanoparticles, as well as relatively homogenous tubular structure, and that the main component of the electrode was $\mathrm{TiO}_{2}$. Cyclic voltammetry (CV) analysis of Ti-based nano electrode result indicated that it was capable of enhanced electrochemical activity in comparison with Ti electrode. It was found that nitrate was removed efficiently by electrochemical means using the Ti-based nano-electrode as cathode with a Ti/Pt anode. The developed system was able to promote the electrochemical reduction of nitrate under a range of experimental conditions. The addition of $\mathrm{NaCl}$ was found to positively affect the removal of by-products, and thus, in combination with nitrate reduction, achieved the goal of clean, safe removal of pollutants.
\end{abstract}

Keywords: Ti-based nano-electrode, Nitrate, Reduction efficiency, Electrochemical approach

\section{$\underline{\text { FULL TEXT }}$}

(C) 2017 The Authors. Published by ESG (www.electrochemsci.org). This article is an open access article distributed under the terms and conditions of the Creative Commons Attribution license (http://creativecommons.org/licenses/by/4.0/). 